\title{
INTERSTELLAR POLARIZATION IN THE SMALL MAGELLANIC CLOUD
}

\author{
C. RODRIGUES ${ }^{1}$, A.M. MAGALHÃES ${ }^{1,2}$, G. COYNE $^{3}$, V. PIIROLA ${ }^{4}$ \\ ${ }^{1}$ Inst. Astron. e Geof., Univ. São Paulo - BRAZIL \\ ${ }^{2}$ Space Astronomy Lab., Univ. Wisconsin - USA \\ ${ }^{3}$ Vatican Obs.Research Group, Univ. Arizona - USA \\ ${ }^{4}$ Obs. and Astrophysics Lab., Univ. Helsinki - FINLAND
}

\begin{abstract}
Data of our on-going program on the interstellar polarization in the Small Magellanic Cloud (SMC) seem to show a correlation between the UV extinction and the parameters of the Serkowski law which describe the polarization. Fits of popular interstellar dust models to the polarization data are not always satisfactory, being better for stars with small wavelength of maximum polarization, $\lambda_{\max }$.
\end{abstract}

\section{Introduction}

Studies of dust in the SMC, mostly based on extinction data, show in the infrared and visible no strong differences to the galactic law and indicate an $R=A_{v} / E(B-V)$ $=2.7 \pm 0.2$ (Bouchet et al, 1985), slightly smaller than the galactic value. The UV extinction is generally characterized by the absence of the $2175 \AA$ bump and shows an extremely steep FUV rise (Prévot et al, 1984). However, one object, AzV456, possesses an extinction similar to the typical galactic law and also its gas-to-dust ratio is typical of the Milky Way(Lequeux et al, 1984). Our aim is to use optical polarization in order to further constrain grain models.

\section{Results}

Magalhães et al (1989) have presented the first wavelength dependent polarization data for the SMC. From that sample, we consider here only the stars whose polarization is large compared to the foreground (galactic) polarization correction. They are AzV211, AzV215, AzV221, AzV398 and AzV456.

Fits to the Serkowski law show that these stars can be divided in two groups. $\mathrm{AzV} 211, \mathrm{AzV} 221$ and $\mathrm{AzV} 398$ show maximum polarization at wavelengths bluer than the galactic average, while AzV215 and AzV456 possess $\lambda_{\max }$ in the $\mathrm{V}$ filter (Table 1). Also, the growth of $\mathrm{K}$ (i.e, the progressive narrowing of the curve) with $\lambda_{\max }$ seems to be faster in the SMC than in the Galaxy. AzV456 presents, as mentioned, a Galactic extinction law while AzV398 shows a 'typical' SMC law. 
Preliminary IUE results for AzV211 (Magalhães et al, 1991) indicate that it does not show the bump as well. Therefore, it seems that the distinct SMC extinctions are related to distinct polarization curves as well.

\section{Conclusion}

The SMC polarization data show typically small $\lambda_{\max }$ values. The absence (or presence) of the $2175 \AA$ extinction bump in the SMC may be correlated with smaller (or larger) $\lambda_{\max }$ values found in our polarization data. It would also seem that the dust models able to reproduce the Galactic interstellar polarization may face difficulties in reproducing some of the polarization curve in the SMC.

\section{Table 1: Fitted Parameters of the Serkowski Law}

\begin{tabular}{lcccc}
\hline Star & $\lambda_{\max }(\mu)$ & $\mathrm{K}$ & $\boldsymbol{P}_{\max }(\%)$ & UV bump \\
\hline 211 & $0.37 \pm 0.22$ & $0.48 \pm 0.67$ & $0.956 \pm 0.089$ & no \\
215 & $0.537 \pm 0.047$ & $2.5 \pm 1.9$ & $0.706 \pm 0.071$ & \\
221 & $0.42 \pm 0.12$ & $1.2 \pm 1.3$ & $0.892 \pm 0.073$ & \\
398 & $0.459 \pm 0.039$ & $1.26 \pm 0.55$ & $1.870 \pm 0.067$ & no \\
456 & $0.572 \pm 0.017$ & $2.06 \pm 0.53$ & $1.189 \pm 0.051$ & yes \\
\hline
\end{tabular}

\section{References}

- Bouchet, P., Lequeux, J., Maurice, E., Prévot, L. and Prévot-Burnichon, M.L. 1985, $A \& A, 149,330$.

- Lequeux, J., Maurice, E., Prévot, L., Prévot-Burnichon, M.L., Rocca-Volmerange B. 1982, $A \& A, 113, \mathrm{L5}$.

- Magalhães, A.M., Piirola, V., Coyne, G.V. and Rodrigues, C.V. 1989, in Interstellar Dust Contributed Papers, eds. A.G.G.M. Tielens and L.J. Allamandola, NASA CP-3036, 347

- Magalhães, A.M., Coyne, G.V., Piirola, V. and Rodrigues, C.V 1991, in preparation.

- Prévot, M.L., Lequeux, J., Maurice, E., Prévot, L. and Rocca-Volmerange, B. 1984, A\&A, $132,389$. 